\title{
The role and relevance of phospholipase D1 during growth and dimorphism of Candida albicans
}

\author{
Bernhard Hube, ${ }^{1,3}$ Daniela Hess, ${ }^{1}$ Carol A. Baker, ${ }^{2}$ Martin Schaller, ${ }^{4}$ \\ Wilhelm Schäfer ${ }^{1}$ and Joseph W. Dolan ${ }^{2}$
}

Author for correspondence: Bernhard Hube. Tel: +49 1888754 2116. Fax: +4918887542328. e-mail: HubeB@ rki.de

1 Institut für Allgemeine Botanik, AMP III, Universität Hamburg, Ohnhorststr. 18, D-22609 Hamburg, Germany

2 Medical University of South Carolina, Department of Microbiology and Immunology, PO Box 250504, Charleston, SC 29425, USA

3 Robert Koch-Institut, NG4, Nordufer 20, D-13353 Berlin, Germany

4 Dermatologische Klinik und Poliklinik der LudwigMaximilians-Universität München, Frauenlobstr.911, D-80337 München, Germany
The phosphatidylcholine-specific phospholipase D1 (PLD1) in Saccharomyces cerevisiae is involved in vesicle transport and is essential for sporulation. The gene encoding the homologous phospholipase D1 from Candida albicans (PLD1) was used to study the role of PLD1 in this pathogenic fungus. In vitro and in vivo expression studies using Northern blots and reverse transcriptasePCR showed low PLD1 mRNA levels in defined media supporting yeast growth and during experimental infection, while enhanced levels of PLD1 transcripts were detected during the yeast to hyphal transition. To study the relevance of PLD1 during yeast and hyphal growth, an essential part of the gene was deleted in both alleles of two isogenic strains. In vitro PLD1 activity assays showed that pld1 mutants produced no detectable levels of phosphatidic acid, the hydrolytic product of PLD1 activity, and strongly reduced levels of diacylglycerol, the product of lipid phosphate phosphohydrolase, suggesting no or a negligible background PLD1 activity in the pld1 mutants. The p/d1 mutants showed no growth differences compared to the parental wild-type in liquid complex and minimal media, independent of the growth temperature. In addition, growth rates of pld1 mutants in media with protein as the sole source of nitrogen were similar to growth rates of the wild-type, indicating that secretion of proteinases was not reduced. Chlamydospore formation was normal in p/d1 mutants. When germ tube formation was induced in liquid media, pld1 mutants showed similar rates of yeast to hyphal transition compared to the wild-type. However, no hyphae formation was observed on solid Spider medium, and cell growth on cornmeal/Tween 80 medium indicated aberrant morphogenesis. In addition, p/d1 mutants growing on solid media had an attenuated ability to invade the agar. In a model of oral candidosis, pld1 mutants showed no attenuation of virulence. In contrast, the mutant was less virulent in two different mouse models. These data suggest that PLD1 is not essential for growth and oral infections. However, they also suggest that a prominent part of the phosphatidic acid and diacylglycerol pools is produced by PLD1 and that the level of these components is important for morphological transitions under certain conditions in C. albicans.

Keywords: PLD1, virulence, signalling pathways, diacylglycerol, phosphatidic acid

\section{INTRODUCTION}

In Saccharomyces cerevisiae a phosphatidylcholine

Abbreviations: DAG, diacylglycerol; FOA, 5-fluoroorotic acid; LPA lysophosphatidic acid; PA, phosphatidic acid; PC, phosphatidylcholine; PITP, phosphatidylinositol transfer protein; PLD, phospholipase D; RHE, reconstituted human epithelium; RT, reverse transcriptase.
(PC)-specific phospholipase D1 (ScPLD1), located in the particulate fraction of haploid and diploid cells (Ella et al., 1995), was found to be essential for sporulation (Rose et al., 1995). This phospholipase had enhanced activity in media with non-fermentable carbon sources and was able to transfer phosphatidate to primary alcohols (Ella et al., 1995). In 1996, a gene encoding a phosphatidylcholine (PC)-specific phospholipase D 
(SPO14) was cloned (Ella et al., 1996; Rose et al., 1995; Waksman et al., 1996). Although spo14 mutants were viable, they were unable to sporulate. ScPLD1deficient mutants were also deficient in growth on nonfermentable carbon sources (Ella et al., 1996; Waksman et al., 1996) and it was postulated that ScPLD1 must play a role in growth, secretion and regulation of membrane lipid synthesis in yeast (Sreenivas et al., 1998; Xie et al., 1998). Both the activity and the proper localization of the enzyme were needed to form prospore walls during sporulation (Rudge et al., 1998). In addition, it was suggested that ScPLD1 generates a pool of phosphatidic acid (PA) or diacylglycerol (DAG) necessary for budding of nascent secretory vesicles from the trans-Golgi network (Xie et al., 1998). It is likely that ScPLD1 plays a dual role in providing lipid components such as PA or DAG, which are necessary for the formation of membranes and secretory vesicles, and may also act as signalling molecules (Rudge et al., 1998). A third potential second messenger, lysophosphatidic acid (LPA), may be produced by the sequential activity of ScPLD1 and a phospholipase A, and may also play important roles in yeast signalling pathways.

Candida albicans is a polymorphic yeast of increasing medical importance. Approximately $90 \%$ of all genes found in C. albicans have a homologous counterpart in the closely related yeast $S$. cerevisiae (Magee \& Scherer, 1998). However, the function of these homologous genes may have changed because C. albicans has long been adapted to growth in the human host. For example, genes encoding components of the MAP kinase mating pathway in $S$. cerevisiae have homologous counterparts in C. albicans. However, in C. albicans this pathway regulates the yeast to hyphal transition (dimorphism), one of the important virulence attributes of C. albicans (Brown \& Gow, 1999; Ernst, 2000). In addition to this pathway, which terminates with the transcriptional activator Cph1 (Liu et al., 1994), at least two additional morphogenetic pathways exist. One of these additional pathways terminates with the transcriptional regulator Efg1 (Stoldt et al., 1997) while the remaining pathway terminates with the transcriptional repressor Tup1 (Braun \& Johnson, 1997). Furthermore, changes in the level of the intracellular second messenger cAMP play an important role in the regulation of dimorphism (Brown \& Gow, 1999; Ernst, 2000).

Since SPO14 is essential for sporulation in S. cerevisiae and sporulation of $C$. albicans has yet to be observed, a homologous PLD1 gene may have a different function in the pathogenic yeast. In fact, a PC-specific PLD activity providing PA, DAG and/or LPA, was shown to be involved in the yeast to hyphal transition (McLain \& Dolan, 1997). The transition was stimulated by the addition of exogenous PLD. Furthermore, the addition of 1-propanol, which resulted in the production of phosphatidylpropanol by PLD1 at the expense of the usual product, phosphatidic acid, delayed hyphal formation. These data indicated that C. albicans possessed a PLD1 gene and that, in addition to the known protein kinase-based signalling pathways, lipid molecules may be involved in the regulation of the dimorphic transition of C. albicans. The aim of this study was, therefore, to study the role and relevance of the gene encoding PLD1 during growth and dimorphism of C. albicans.

\section{METHODS}

C. albicans and Escherichia coli strains. The $\mathrm{Ura}^{-}$strain CAI4 of C. albicans (Robin) Berkhout, used for disruption of PLD1, and the ura3::imm434 $\mathrm{Ura}^{+}$(heterozygote of CAI4) CAF2-1 were kindly provided by $\mathrm{Dr} \mathrm{W}$. A. Fonzi, University of Washington, USA. Growth rates, dimorphism and phospholipase assays of pld1::hisG $\mathrm{Ura}^{+}$isogenic mutants were compared with the parental $\mathrm{Ura}^{+}$clinical isolate SC5314 (Gillum et al., 1984), the heterozygote CAF2-1, and/or the morphogenic mutants cph1:: his $G \mathrm{Ura}^{+}$, efg1:: his $G \mathrm{Ura}^{+}$and cph1::hisG/efg1::hisG $\mathrm{Ura}^{+}$(kindly provided by Dr G. R. Fink, Massachusetts Institute of Technology, USA and Dr J. Ernst, University of Düsseldorf, Germany). In addition, strain B311 (Vasquez-Torres et al., 1999) was used for virulence studies. E. coli XL-Blue (Stratagene) was used for transformation of cloned PLD1 fragments.

Plasmids and fosmid library. pBluescript KS $(+/-)$ (Stratagene) was used for subcloning of PLD1 fragments. The fosmid library (Magee \& Scherer, 1998) was kindly provided by Dr S. Scherer, University of Minnesota, USA.

Media and growth conditions. To investigate PLD1 gene expression in SC5314 and growth or hyphal formation of pld1:: his $G \mathrm{Ura}^{+}$mutants, we used liquid YPG, minimal and protein medium (Hube et al., 1994). For hyphal growth, cells were suspended in $5 \%(\mathrm{v} / \mathrm{v})$ calf serum (Gow \& Gooday, $1982)$, or induced by the addition of $N$-acetylglucosamine (Mattia et al., 1982) or the regime of $\mathrm{pH} /$ temperatureregulated yeast to hyphal transition was used (Buffo et al., 1984). To perform growth tests on solid media, C. albicans cells were grown overnight in YPG and diluted to $2 \times 10^{7}$ cells $\mathrm{ml}^{-1}$. Five microlitres of this suspension and $5 \mu \mathrm{l}$ serial dilutions of each strain were spotted onto each type of plate (YPG, minimal medium, protein medium), and the plates were incubated at $25^{\circ} \mathrm{C}, 37^{\circ} \mathrm{C}$ and $42^{\circ} \mathrm{C}$ for at least $2 \mathrm{~d}$. For hyphal induction on solid media, cells were streaked onto Spider agar (Liu et al., 1994) and cornmeal agar with $1 \%$ Tween 80 (CMA/T) (Buckley et al., 1982) and incubated at $37^{\circ} \mathrm{C}$. For chlamydospore formation, cells were streaked on Spider agar or CMA/T at low concentrations, covered with coverslips and incubated at $25^{\circ} \mathrm{C}$ for several days. Colonies were photographed using a Zeiss KF2 light microscope equipped with a Polaroid MicroCam camera. Pictures of representative colonies were taken after $24 \mathrm{~h}$ on CMA/T and after $72 \mathrm{~h}$ on Spider agar. Following photography, the plates were washed vigorously with water to remove cells on the surface of the agar. The plates were allowed to dry at room temperature for $1 \mathrm{~h}$ prior to the second round of photography.

PCR. PCR was used to prepare the disruption cassette and hybridization probes for Southern and Northern blots, and to confirm the disruption of the PLD1 gene. The following pairs of primers were used to amplify hybridization probes of the ORF of SPO14: ScPLD1-1 (5'-TGTCGTTATTGATGAAACAT-3') and ScPLD1-2 (5'-TTATCAAGTCGGTGTCTCTA-3'); ScPLD1-3 (5'-CATCAATAACGACAAACTTCTCG-3') and ScPLD1-4 (5'-CGTCGCGTTCCAACAACTCAC$\left.3^{\prime}\right)$; ScPLD1-5 (5'-TAGAGACACCGACTTGATAAAG-3'); and ScPLD1-6 (5'-TCCAGTGAACCATCATCTAG-3'). The following primers were used to amplify fragments of the ORF of PLD1 PLD1-1 (5'-GACCAACGCATCACCAATTC-3'); 
PLD1-2 (5'-CAGCTTGTTTCATCGACGG-3)' ; PLD1-3 (5'AGCTGCCATATATGGCTTACC-3'); PLD1-4 (5'-GACAGCACTAAGAGTGGCAG-3'); PLD1-5 (5'-GAATGAGGTTGATGAGAGAGC-3') ; PLD1-6 (5'-CAAAGATACAGTAGGGAACTC-3').

RNA isolation. For Northern analysis, total RNA from C. albicans was prepared as described by Hube et al. (1994). For reverse transcriptase (RT)-PCR, total RNA was isolated using RNAPure (Peqlab Biotechnologie) according to the manufacturer's instructions.

Northern and Southern analyses. Northern blot analysis was performed as described by Hube et al. (1994). For Southern blot analysis of genomic or fosmid DNA, standard protocols were used (Sambrook et al., 1989). Southern blots and the fosmid library were hybridized to identify PLD1 using a nonradioactive digoxigenin (DIG)-labelling kit (Boehringer Mannheim). Three PCR-generated probes of SPO14 were used. Probe A was amplified with ScPLD1-1 and ScPLD1-2, and contained a $1000 \mathrm{bp}$ fragment of the SPO14 ORF encoding the conserved boxes 4, 5 and 7 (Waksman et al., 1996). Probe B was amplified with ScPLD1-3 and ScPLD1-4, and contained a $1100 \mathrm{bp} \mathrm{SPO14}$ fragment located 5' of probe A, and probe $\mathrm{C}$ was amplified with ScPLD1-5 and ScPLD1-6, and contained a $1380 \mathrm{bp}$ SPO14 fragment located $3^{\prime}$ of probe A. To analyse PLD1 gene disruption, a 430 bp long T7 (Stratagene) PLD1-2 PCR fragment of plasmid pHPLD1 or the random labelled his $G:$ :URA3: : his $G$ cassette of pMB7 was used as probe. For Northern blots, the same fragment of subclone pHPLD1 and a $700 \mathrm{bp}$ PCR fragment of TEF3 (Colthurst et al., 1992; Hube et al., 1994) were labelled with $\left[\alpha^{32} \mathrm{P}\right] \mathrm{dCTP}$ ( $3000 \mathrm{Ci} \mathrm{mmol}^{-1} ; 111 \mathrm{TBq} \mathrm{mmol}^{-1}$ ) (Amersham). mRNA levels were measured relative to the rRNAs by loading approximately equal amounts of total RNA in each lane of the Northern blots. In addition, the TEF3 mRNA (Colthurst et al., 1992) was probed as a positive (non-quantitative) control (Hube et al., 1994).

For Southern blots, membranes were hybridized without formamide using digoxigenin-labelled probes and washed as described by the manufacturer (Boehringer Mannheim) with either $65^{\circ} \mathrm{C}$ (low stringency) or $68^{\circ} \mathrm{C}$ (high stringency) incubation temperatures.

Screening of the fosmid library. The fosmid library was blotted onto nylon membranes using a vacuum dot-blot system (Schleicher \& Schuell). E. coli cells were lysed and fosmid DNA was denatured with $0.5 \mathrm{M} \mathrm{NaOH}, 1.5 \mathrm{M} \mathrm{NaCl}$. After neutralization with $1 \mathrm{M}$ Tris $/ \mathrm{HCl} \mathrm{pH} 8 \cdot 0,1.5 \mathrm{M} \mathrm{NaCl}$, fosmid DNA was fixed by baking and membranes were hybridized as described for Southern analysis. DNA from positive fosmids was isolated as described by Hube et al. (2000).

DNA sequencing. DNA subcloned into pBluescript was sequenced by Seqlab Laboratories or MWG.

RT-PCR. One microgram of DNase I-treated total RNA was used for cDNA synthesis as described by Schaller et al. (1998). The cDNA was purified using NucleoSpin extract columns (Macherey \& Nagel). To detect PLD1 transcripts, primers PLD1-5 and PLD1-6 were used to amplify an 889 bp cDNA fragment of PLD1. To prove the absence of contaminating genomic DNA, we used primers specific for the introncontaining gene encoding elongation factor 1 (EFB1) (Maneu et al., 1996). Using primers EFB5' (5'-ATTGAACGAATTCTTGGCTGAC-3') and EFB3' (5'-CATCTTCTTCAACAGCAGCTTG-3'), a 916 bp PCR fragment was amplified when genomic DNA was present. In contrast, a 551 bp sized RTPCR fragment of the EFB1 transcript which does not contain an intron of $365 \mathrm{bp}$ in size was amplified when cDNA was used as a template. Even traces of DNA were detectable, when EFB5' and the intron-specific primer EFBint (5'-TCTTGAGGCCACCTCATAAAC-3') were used. In these control experiments an additional 264 bp fragment was amplified.

Candida transformation and gene disruption. Protoplasts were prepared and PLD1 disruption cassettes were transformed as described by Hube et al. (1997). For 5-fluoroorotic acid (FOA) selection of $\mathrm{Ura}^{-}$recombinants (Fonzi \& Irwin, 1993; Gow et al., 1994), cloned transformants were resuspended in $1 \mathrm{ml}$ water and plated on SD agar containing FOA $\left(0.01 \mathrm{~g} \mathrm{ml}^{-1}\right)$ and uridine $\left(25 \mu \mathrm{g} \mathrm{ml}^{-1}\right)$.

To construct a disruption cassette, pHPLD1 (Fig. 1b) was digested with $S a c$ I and SalI, which removes a 2075 bp internal fragment. Since this fragment was 450 bp longer than expected from the published sequence (Kanoh et al., 1998), we sequenced this part of the gene and found a $\mathrm{T}$ instead of a $\mathrm{C}$ in position 1266, which created a second $S a c$ site at $1262 \mathrm{bp}$. The removed fragment contained sequences encoding the HXKXXXXD, HKD and GGGR motifs, which are critical for activity of ScPLD1 in S. cerevisiae (Waksman et al., 1996). The his $G:$ :URA3:: hisG cassette of pMB7 (Fonzi \& Irwin, 1993) was removed by SacI/SalI digestion, and ligated into pHPLD1 to give pHP1ura (Fig. 1b). pHP1ura was linearized with PvuII and transformed into CAI4. Integration of the cassette into the PLD1 locus was confirmed by Southern analysis for each step of the disruption procedure (Fig. 1c, d). When genomic DNAs of first round transformants were digested with EcoRI and hybridized to the PLD1 probe, wild-type alleles were $>12 \mathrm{~kb}$ in size (Fig. 1c). Alleles which contained the his $G:: U R A 3:$ : his $G$ cassette showed two bands, one with a size similar to the wild-type band and a second band of $3.5 \mathrm{~kb}$. The correct integration could be confirmed using BamHI, which again produced a second band of $3.5 \mathrm{~kb}$ in addition to the large band when hybridized to the PLD1 probe. EcoRI and Bam HI digests of DNA from FOA-resistant segregants showed a single band $1 \mathrm{~kb}$ smaller than the size of the wild-type band when hybridized with the PLD1 probe. Since it was difficult to distinguish between the wild-type band and the large bands of disrupted alleles, Southern analysis was repeated using a his $G:$ :URA3 probe to confirm the disruption of both alleles (Fig. 1d). In first round transformants a $9 \mathrm{~kb}$ and a $3.5 \mathrm{~kb}$ fragment of the disrupted allele hybridized in EcoRI digests as expected. In FOA segregants only one band $>9 \mathrm{~kb}$ containing the remaining his $G$ part hybridized. In second round transformants the larger band $>9 \mathrm{~kb}$, the $9 \mathrm{~kb}$ band and the 3.5 EcoRI band were all visible, confirming that both alleles were disrupted and no additional integration had occurred. Using the two primers pHPLD1-3 and pHPLD1-4, his G integration and disruption of both alleles in two isogenic strains was confirmed by PCR (not shown). The two isogenic pld1:: hisG/pld1:: hisG::URA3:: hisG null mutants $\left(\mathrm{Ura}^{+}\right.$)

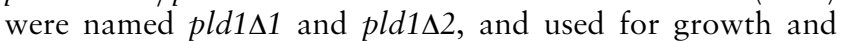
activity tests.

PLD1 assays. Cell extracts were prepared and PLD1 activity was assayed as described by McLain \& Dolan (1997).

Experimental infection. Virulence properties of pld1 mutants were tested in an in vitro model of oral candidosis based on reconstituted human epithelium (RHE) as described by Schaller et al. (1998). RHE was infected with $2 \times 10^{6}$ C. albicans wild-type or mutant cells in $50 \mu \mathrm{l}$ PBS. Controls contained $50 \mu \mathrm{l}$ PBS alone. Inoculated and uninoculated 
(a) Genomic locus

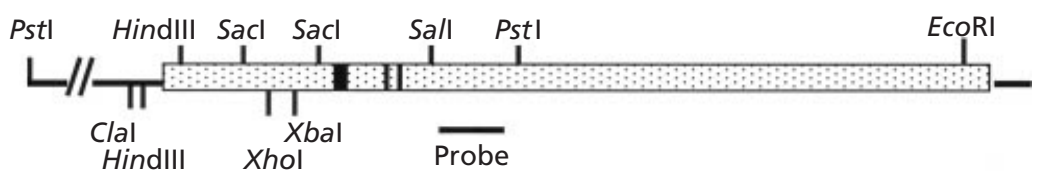

$1 \mathrm{~kb}$

(b) Deletion of disruption fragment

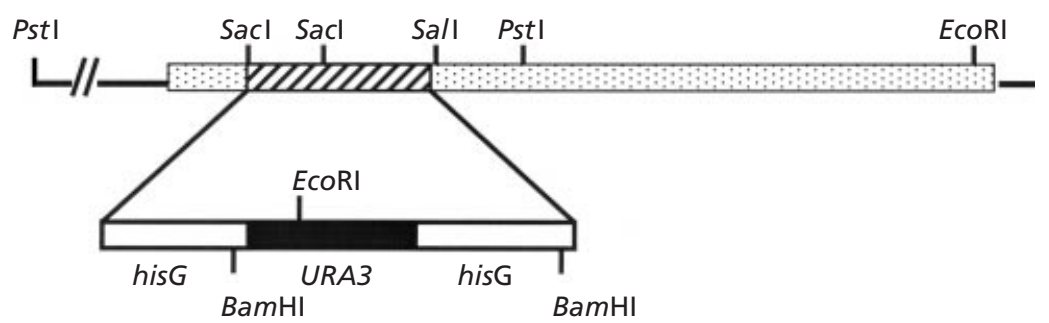

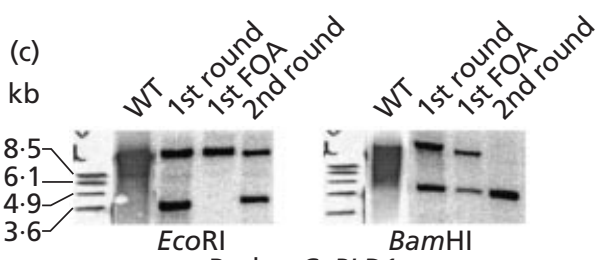

Probe: CaPLD1

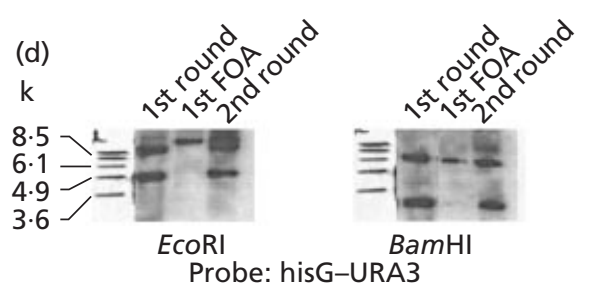

Fig. 1. Restriction map of the genomic locus of PLD1 (a), the construction of pHP1ura (b) and Southern analysis of PLD1 transformants (c,d). The 5130 bp ORF of PLD1 (dotted box) and important restriction sites are shown in (a). The Pstl fragment was cloned in pHPLD1 and used to produce the disruption cassette. The HXKXXXXD, HKD and GGGR motifs (small black boxes) are located between the Sall and the second Sacl site of the ORF. An additional Sacl site was identified at the $5^{\prime}$ end. This essential Sacl-Sall fragment was replaced by the hisG::URA3:: hisG cassette introducing a new EcoRI site in the disrupted ORF. Restriction digests of the wild-type CAI4, the first transformant, first FOA-segregant and second transformant (p/d1 11 ) were hybridized with the PLD1 (c) or the hisG::URA3 probe (d). The same results were obtained for a second isogenic mutant (p/d1 $\Delta 2$ ). See text for further details.

cultures were incubated at $37^{\circ} \mathrm{C}$ with $5 \% \mathrm{CO}_{2}$ at $100 \%$ humidity for $12-48 \mathrm{~h}$.

Animal studies. Germ-free immunodeficient beige nude $(\mathrm{bg} /$ bg $n u / n u)$ mice (Fodstad et al., 1984) were inoculated orally with approximately $1 \times 10^{6}$ c.f.u. of either wild-type SC5314 or pld1 11 mutant cells; a second wild-type strain, B311, was also used for comparison (Vasquez-Torres et al., 1999). Viability was monitored for 6 weeks post-inoculation. Germfree immunodeficient transgenic $\varepsilon 26$ mice (Wang et al., 1994) were inoculated orally with approximately $1 \times 10^{6}$ c.f.u. wildtype SC5314, wild-type B 311 or pld1 11 mutant cells. Viability was again monitored for 6 weeks post-inoculation. Survival curves were calculated according to the method of KaplanMeier using GraphPad Prizm 3.0a software and were compared using the log rank test of Mantel-Haenszel.

\section{RESULTS}

\section{Cloning of PLD1}

To identify genes in C. albicans homologous to SPO14, genomic DNA of SC5314 was digested with EcoRI and blotted onto membranes. The blot was hybridized with SPO14 probes and four distinct bands between 3 and $10 \mathrm{~kb}$ were identified, indicating that homologous sequences exist in the genome of C. albicans. The same probes were used to screen 10 membranes of a fosmid library, each containing 96 fosmid clones (Magee \& Scherer, 1998). Two positive clones, 2G2 and 6E4 (http://alces.med.umn.edu/candida/fosmidinfo.html) were further analysed. Each clone contained a $7.5 \mathrm{~kb}$
Pst I and a 9.0 kb EcoRI fragment. The $7.5 \mathrm{~kb}$ fragment was cloned into the PstI site of pBluescript $\mathrm{KS}(+/-)$ to give pHPLD1. Sequencing from both ends revealed ORFs. Sequences from the T3 site had homologies to several hypothetical GTPase genes with the highest similarity to an ORF of $S$. cerevisiae with unknown function (accession no. U51032). The opposite strand of the T7 site encoded an amino acid sequence with high similarity (40\% identity) to ScPLD1 corresponding to amino acids $473-826$ of the deduced protein. The DNA sequence of this region of the Pst I fragment was found to be $98 \%$ identical to the PLD1 sequence published by Kanoh et al. (1998) and localized on contig numbers Con4-1904 and Con4-2834 of the Stanford genome project website (http://www-sequence.stanford.edu/ group/candida/index.html; gnl|Stanford-5476| C.albicans-Con4-1904 Candida albicans unf... 7002 0.0; gnl|Stanford-5476|C.albicans-Con4-2834 Candida albicans unf... 3251). Since fosmid 6E4 was mapped to chromosome 1, PLD1 must be located on this chromosome.

\section{PLD1 in vitro expression studies}

Since PLD1 activity was stimulated by inducers of the dimorphic transition (McLain \& Dolan, 1997), we questioned whether PLD1 is regulated during morphogenesis. Hyphal formation of C. albicans was induced by $\mathrm{pH} /$ temperature shift in Lee's medium (Buffo et al., 1984). Cell samples were taken 60, 90, 120, 180 

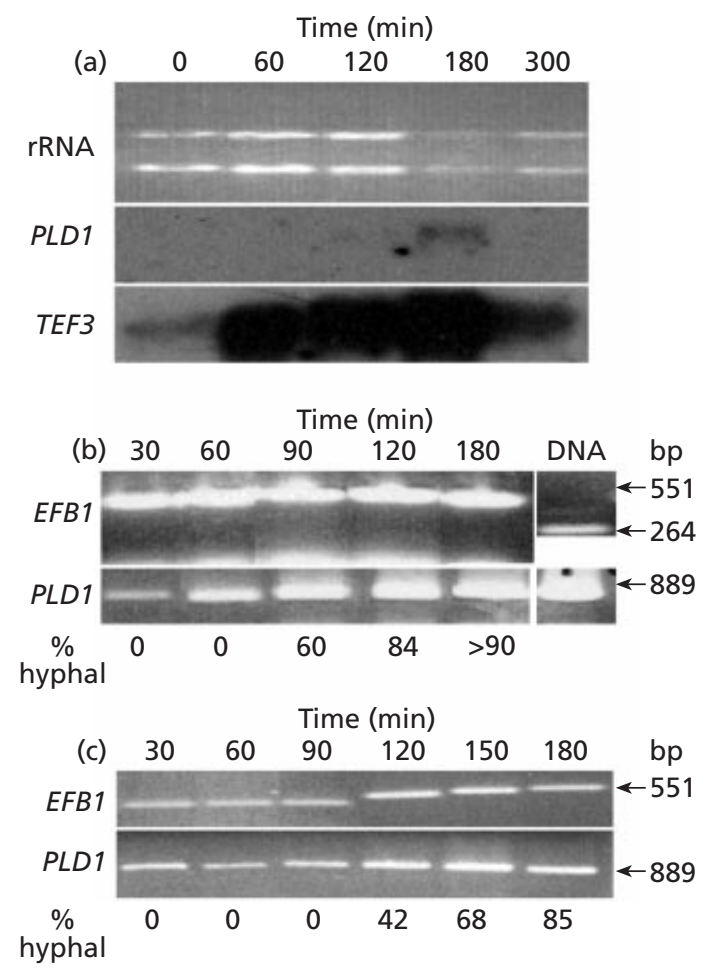

Fig. 2. Expression of PLD1 during yeast to hyphal transition in $C$. albicans shown by Northern analysis (a) and RT-PCR (b, c). Total RNA of pH/temperature induced cells was analysed in (a) and (b). Expression of PLD1 in $\mathrm{N}$-acetylglucosamine induced cells is shown in (c). The housekeeping genes TEF3 and EFB1 were used as internal controls (Hube et al., 1994; Schaller et al., 1998). mRNA levels were measured relative to the rRNAs by loading approximately equal amounts of total RNA in each lane of the Northern blots. In addition, the TEF3 mRNA (Colthurst et al., 1992) was probed as a positive (non-quantitative) control (Hube et al., 1994). For RT-PCR, the EFB1 primers amplifying the intron-containing region were used to show the absence of contaminating DNA. EFB1 cDNA revealed a 551 bp fragment. Only traces of DNA were detectable when primer EFB5' and the intron-specific primer EFBint were used. In these control experiments an additional $264 \mathrm{bp}$ fragment was amplified when DNA was present.

and $300 \mathrm{~min}$ after induction, hyphal formation was monitored and total RNA was isolated. After $60 \mathrm{~min}$, no germ tube formation was seen; after $90 \mathrm{~min}$, germ tube formation was $57 \%, 83 \%$ after $120 \mathrm{~min}$ and $>90 \%$ after $180 \mathrm{~min}$. The RNA was used to measure PLD1 transcripts by Northern analysis and RT-PCR (Fig. 2). Northern analysis showed expression of PLD1 at $180 \mathrm{~min}$, but no signals in the other samples (Fig. 2a). In contrast, the more sensitive RT-PCR analysis showed a continuous up-regulation of PLD1 expression during the dimorphic transition (Fig. 2b). A similar expression pattern was seen when the experiment was repeated. To show that the up-regulation was due to the morphological transition, hyphal formation was also induced by the addition of $N$-acetylglucosamine (Mattia et al., 1982). After $120 \mathrm{~min}, 42 \%$ of the cells produced germ tubes. After 150 and $180 \mathrm{~min}, 68 \%$ and $85 \%$ of the cells

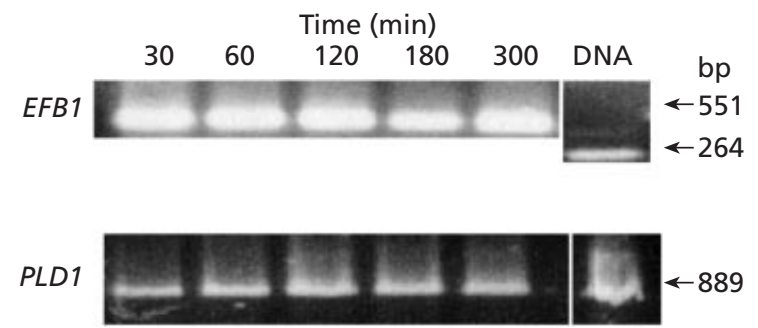

Fig. 3. Continuous low level expression of PLD1 during growth in medium containing BSA as the sole source of nitrogen as determined by RT-PCR.

showed hyphal formation. Expression of PLD1 was observed during the course of germ tube production (Fig. 2c). Similar results were obtained when this experiment was repeated or when hyphal formation was induced by the addition of serum (not shown).

In $S$. cerevisiae it has been postulated that ScPLD1 may play a role in growth, secretion and regulation of lipid biosynthesis (Sreenivas et al., 1998). Therefore, we studied the expression of PLD1 in medium with protein as the sole source of nitrogen. Under these conditions high levels of secreted aspartic proteinases must be secreted to support growth, while cells unable to secrete distinct Saps do not grow (Hube et al., 1997). C. albicans cells were grown in protein medium and PLD1 expression was monitored 30,60,120, 180 and $300 \mathrm{~min}$ after inoculation using RT-PCR (Fig. 3). A continuous low-level expression of PLD1 was observed in two independent experiments. In addition, YPD precultures without proteinase-inducing BSA also showed PLD1 expression (not shown).

\section{PLD1 is expressed during experimental infections}

To investigate whether PLD1 is expressed during infections with $C$. albicans, we screened for mRNA transcripts in infected artificial tissue. A model of oral candidosis based on RHE (Schaller et al., 1998) was used to study PLD1 expression during experimental infection. Total RNA was isolated from infected RHE at different time points during the course of an infection and mRNA transcripts were amplified by RT-PCR using primers PLD1-5 and PLD1-6. PLD1 transcripts were detectable 12,36 and $48 \mathrm{~h}$ after infection, indicating that PLD1 is expressed during an infection process.

\section{PLD1 encodes a phospholipase D}

Expression studies showed a low-level expression of PLD1 under most conditions and an up-regulation during hyphal formation. PLD1 activity has also been shown to increase during morphogenesis (McLain \& Dolan, 1997). To investigate the relevance of PLD1 during growth and dimorphism, we produced mutants that lack functional copies of PLD1 (Fig. 1). In these 


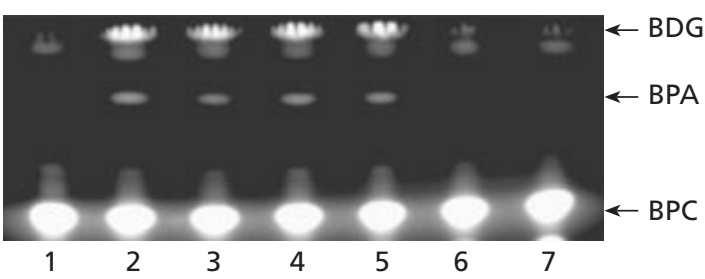

Fig. 4. pld1 null mutants lack detectable PLD1 activity. Cell extracts from the wild-type (CAF2-1), pld1 $\Delta 1$ and $p / d 1 \Delta 2$ strains were assayed for PLD1 activity with the fluorescent in vitro assay (McLain \& Dolan, 1997). The sample in lane 1 contained no cell extract; lane 2 contained cell extract from efg 1 ; lane 3 contained cell extract from cph1; lane 4 contained cell extract from efg 1/cph1; lane 5 contained cell extract from wild-type strain CAF2-1; lane 6 contained cell extract from pld1 $\Delta 1$ and lane 7 contained cell extract from pld1 22 . BPC, BODIPYphosphatidylcholine; BPA, BODIPY-phosphatidic acid; BDG, BODIPY-diglyceride.

mutants, an essential part of each allele of the PLD1 gene was removed in two isogenic strains.

Sequence alignment of PLD1 with SPO14 and other phospholipase D genes strongly suggested that PLD1 encodes a phospholipase D. Southern analysis of wildtype and pld1 null mutants and sequence comparison with sequence data from the Candida genome project (http://www-sequence.stanford.edu/group/candida) suggested that PLD1 exists as a single copy gene and that C. albicans does not contain additional homologous PLD genes. However, additional heterologous PLD genes may exist in S. cerevisiae (Mayr et al., 1996; Waksman et al., 1997). To prove whether PLD1 encodes phospholipase D1 and whether this is the only gene responsible for PLD1 activity in C. albicans, we measured PLD activity using a fluorescent analogue of PC (BODIPY-phosphatidylcholine; Molecular Probes) in extracts from wild-type and the pld1 null mutants. No detectable BODIPY-phosphatidic acid and strongly reduced levels of BODIPY-diglyceride were detected in both pld1 11 and $p l d 1 \Delta 2$ when compared with the wildtype SC5314 or CAF2-1 (Fig. 4), indicating that PLD1 encodes the major or only PC-specific phospholipase D in C. albicans. Fig. 4 also demonstrates that basal PLD1 activity is unaffected by mutations in CPH1, EFG1 or both.

\section{Growth of pld1 null mutants}

To test the effect of the lack of PLD1 activity on cell growth of $C$. albicans, several growth conditions were tested. Since expression of PLD1 was observed in complex media, we first analysed the growth of $p l d 1 \Delta 1$ and pld1 12 in YPG medium. No differences in growth rates were observed at $25^{\circ} \mathrm{C}, 37^{\circ} \mathrm{C}$ or $42^{\circ} \mathrm{C}$. The same results were observed when the pld1 mutants were grown in minimal medium with protein as the sole source of nitrogen at $37^{\circ} \mathrm{C}$, indicating that sufficient proteinases were secreted and secretion was not reduced significantly due to the lack of PLD1 activity.

\section{Hyphal formation of pld1 null mutants in liquid media}

Expression of PLD1 was observed during the yeast to hyphal transition when cells were induced by $\mathrm{pH} /$ temperature shift, $\mathrm{N}$-acetylglucosamine or serum. To study the relevance of PLD1 during the dimorphic transition, the pld 1 mutants were induced and the rate of hyphal formation was compared with the wild-type strain SC5314. No differences were observed in all cases. For example, in serum-induced cultures both wild-type and mutant cells had more than $50 \%$ germ tubes after $60 \mathrm{~min}$ and more than $90 \%$ hyphal cells after $120 \mathrm{~min}$.

\section{Chlamydospore production}

C. albicans has the ability to form thick-walled cells, termed chlamydospores, which arise on elongated suspensor cells situated on pseudohyphal or hyphal cells. Although they do not appear to allow long term survival, the morphology resembles true yeast spores. S. cerevisiae spo14 mutants were viable, but unable to sporulate. Therefore, we investigated the role of PLD1 during chlamydospore formation. Chlamydospore formation was induced on Spider agar and CMA/T under microaerobic conditions at $25^{\circ} \mathrm{C}$. Although suspensor cells of pld1 mutants sometimes appear to be more swollen compared to wild-type suspensor cells, both pld1 mutants were able to produce chlamydospores to the same extent as the wild-type.

\section{Morphological defects of pld1 null mutants on solid media}

Although no differences in hyphal formation were seen in liquid media, there were substantial differences on solid media. On solid serum agar, an overall reduction of hyphal formation was seen for the pld1 mutants when compared to the wild-type. On Spider agar, pld1 mutants failed to make hyphae that radiate away from the central colony, a phenotype previously reported for cph1 (Liu et al., 1994) and efg1 (Stoldt et al., 1997) mutants (Fig. 5). The pld1 mutants produced hyphae that penetrated into the agar under the colony, but the extent of penetration was reduced relative to that seen with the wild-type or cph1 mutant. The hyphal formation of the wild-type is variable from one plate to another, making absolute quantitations impossible. Nevertheless, the majority (greater than $50 \%$ ) of wildtype colonies formed radiating hyphae on Spider medium while the pld1 mutants failed to form any colonies with radiating hyphae. The phenotype on CMA/T medium was most pronounced after the first $24 \mathrm{~h}$ growth. The number of hyphae radiating from the pld1 mutant colonies (Fig. 5h, i) was substantially greater than the number radiating from either the wild-type (Fig. 5g) or the cph1 mutant (Fig. 5j). As with Spider medium, the wild-type colonies exhibited some degree of variability. Nevertheless, greater than $75 \%$ of wildtype colonies gave rise to fewer than 10 radiating hyphae after $24 \mathrm{~h}$ at $37^{\circ} \mathrm{C}$ with most producing only one or two 


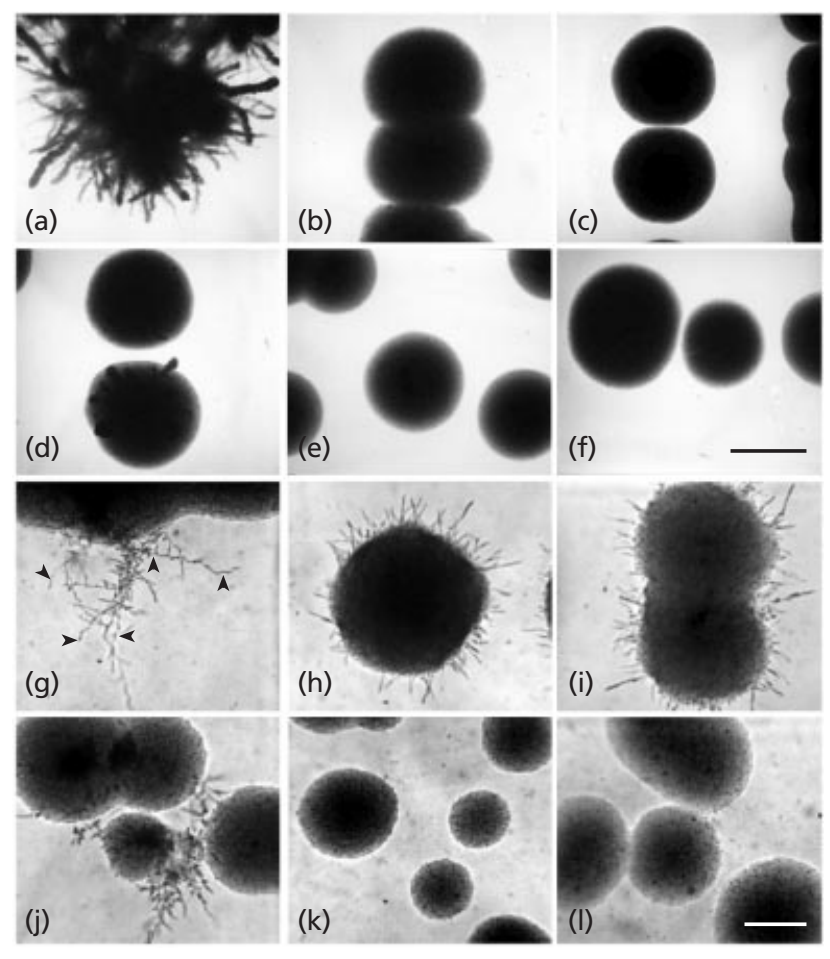

Fig. 5. Phenotype of mutants on inducing media. Cells were streaked onto Spider agar $(\mathrm{a}-\mathrm{f})$ and $\mathrm{CMA} / \mathrm{T}(\mathrm{g}-\mathrm{I})$ and incubated at $37{ }^{\circ} \mathrm{C}$. Strains used were CAF2-1 $(\mathrm{a}, \mathrm{g})$, pld $1 \Delta 1(\mathrm{~b}, \mathrm{~h}), \mathrm{p} / \mathrm{d} 1 \Delta 2$ $(c, i)$, cph1 (d, j), efg1 (e, k) and cph1/efg1 double mutant (f, I). Colonies on Spider agar were magnified $20 \times$ and the colonies on CMA/T were magnified $80 \times$. The arrowheads in $(\mathrm{g})$ indicate kinks in hyphae that are common to wild-type hyphae but essentially absent from the p/d1 hyphae. Bars, $1 \mathrm{~mm}(\mathrm{f}) ; 0.2 \mathrm{~mm}$ (l). Parts $(a-f)$ and $(g-l)$ have the same magnifications.

such hyphae. In contrast, all pld 1 colonies gave rise to numerous hyphae (20-50 hyphae per colony) radiating from the central colony. Furthermore, the hyphae produced by the pld 1 mutants were straighter and shorter than those produced by the wild-type or cph1 colonies, which were long and kinked (marked with arrowheads in Fig. 5).

S. cerevisiae cells exhibit a haploid-specific ability to invade agar and this process is regulated by components of the pheromone response pathway (Roberts \& Fink, 1994; Cook et al., 1996). Homologues of some of these components regulate morphogenesis in C. albicans; therefore, the ability of wild-type and pld 1 mutant cells to invade the agar was also examined (Fig. 6). Cells within the agar more clearly revealed the differences in the ability of the strains to invade a solid surface and in the pattern of lateral budding along the hyphae. The wild-type cells and the $c p h 1$ mutant robustly invaded the agar of Spider medium, producing hyphae that were heavily decorated with yeast-form cells (Fig. 6a, c). The efg1 mutant cells had a reduced ability to penetrate the surface of Spider medium and failed to form hyphae of any substantial length (Fig. 6d). The pld1 mutants were able to invade Spider agar, but failed to form lengthy

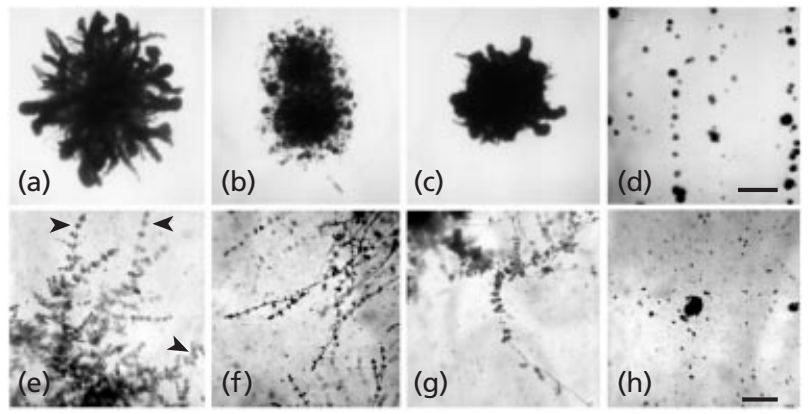

Fig. 6. Mutants have a distinct agar-invasion phenotype. Washed plates were photographed to examine the morphologies of hyphae within the agar medium. (a-d) Cells on Spider agar; $(e-h)$ cells on CMA/T. The strains examined were CAF2-1 (a, e), pld1 $\Delta 1$ (b, f), cph1 (c, g) and efg1 (d, h). The arrowheads in (e) indicate hyphae decorated with large clusters of cells that are common to wild-type hyphae but essentially absent from the pld hyphae. Bars, $1 \mathrm{~mm}$ (d); $0.2 \mathrm{~mm}$ (h). Parts $(a-d)$ and $(e-h)$ have the same magnifications.

hyphae and the hyphae that did form were not as heavily decorated by yeast-form cells (Fig. 6b). Differences in lateral budding were also seen with cells growing on cornmeal agar in which wild-type and cph1 cells formed clumps of yeast cells at regular intervals along the hyphae (Fig. 6e, g). The pld1 mutants formed small clusters containing only a few cells per cluster (Fig. 6f). As with Spider medium, the efg1 mutant cells were not able to invade the agar efficiently and produced only foci of cells just below the surface (Fig. 6h). The relevance of these defects to growth in vivo is not known at this time.

\section{Null mutants exhibit reduced virulence}

Since we showed that the PLD1 gene is expressed in wild-type strains during infection of our model of oral candidosis (RHE), we infected the RHE tissue with the pld1 null mutants and compared the phenotype with the parental wild-type strain. Histological samples were taken $12 \mathrm{~h}$ and $36 \mathrm{~h}$ after infection and 60 sections were analysed for each time point and each strain. No obvious differences were observed between the wild-type and mutant strains.

Mutations in several genes regulating morphogenesis in C. albicans share the same phenotype on solid Spider medium as the pld 1 mutants. These other mutants also exhibit reduced virulence in animal models of infection compared to the parental strain SC5314. The lethality of the pld1 11 mutant was assessed in a mouse model utilizing $b g / b g n u / n u$ immunodeficient mice (Fodstad $e t$ al., 1984). For this experiment, an additional wild-type strain of C. albicans was used. B311 and SC5314 are independently isolated wild-type strains. Strain B311 has been used extensively in animal studies, including studies with the $b g / b g n u / n u$ mice, and was included solely to provide a point of reference to earlier virulence studies with this strain of mice. The survival of mice orally inoculated with $10^{6}$ c.f.u. was followed for up to 
Table 1. Lethality of C. albicans strains in $\mathrm{bg} / \mathrm{bg} \mathrm{nu} / \mathrm{nu}$ mice

Mice were inoculated orally with $1 \times 10^{6}$ c.f.u. of the indicated strain of C. albicans. Differences between wild-type and pld $1 \Delta 1$ curves were significant with $P$ values of 0.0002 (B322 vs.

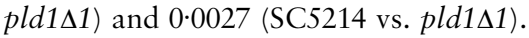

\begin{tabular}{|lcrc|}
\hline \multirow{2}{*}{$\begin{array}{l}\text { Candida } \\
\text { strain }\end{array}$} & $\begin{array}{c}\text { Time post- } \\
\text { inoculation }(\mathrm{d})\end{array}$ & \multicolumn{2}{c|}{ Survival } \\
\cline { 3 - 4 } & & Percentage & $\begin{array}{c}\text { No. alive/ } \\
\text { no. dead }\end{array}$ \\
\hline B311 & 14 & 100 & $12 / 0$ \\
& $21-28$ & 58 & $7 / 5$ \\
& $35-42$ & 0 & $0 / 12$ \\
SC5314 & 14 & 100 & $4 / 0$ \\
& $21-28$ & 0 & $0 / 4$ \\
& $35-42$ & 0 & $0 / 4$ \\
pld1 & 14 & 100 & $6 / 0$ \\
& $21-28$ & 100 & $6 / 0$ \\
& $35-42$ & 100 & $6 / 0$ \\
\hline
\end{tabular}

Table 2. Lethality of C. albicans strains in tg\&26 mice

Mice were inoculated orally with $1 \times 10^{6}$ c.f.u. of the indicated strain of C. albicans. Differences between wild-type and pld $1 \Delta 1$ curves were significant with $P$ values of $<0.0001$ (B311 vs.

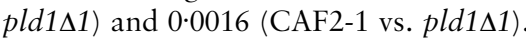

\begin{tabular}{|lcrc|}
\hline \multirow{2}{*}{$\begin{array}{l}\text { Candida } \\
\text { strain }\end{array}$} & $\begin{array}{c}\text { Time post- } \\
\text { inoculation }(\mathrm{d})\end{array}$ & \multicolumn{2}{c|}{ Survival } \\
\cline { 3 - 4 } & & Percentage & $\begin{array}{c}\text { No. alive/ } \\
\text { no. dead }\end{array}$ \\
\hline B311 & 14 & 100 & $17 / 0$ \\
& $21-28$ & 18 & $3 / 14$ \\
& $35-42$ & 0 & $0 / 17$ \\
CAF2-1 & 14 & 0 & $0 / 5$ \\
& $21-28$ & 0 & $0 / 5$ \\
& $35-42$ & 0 & $0 / 5$ \\
pld1 101 & 14 & 100 & $6 / 0$ \\
& $21-28$ & 100 & $6 / 0$ \\
& $35-42$ & 100 & $6 / 0$ \\
\hline
\end{tabular}

$42 \mathrm{~d}$ post-inoculation (Table 1 ). As expected, both wildtype strains of Candida were $100 \%$ lethal to the immunodeficient mice with the death of all inoculated animals within $42 \mathrm{~d}$ post-inoculation. In contrast, all

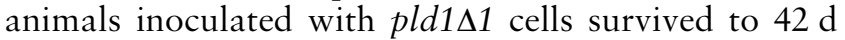
post-inoculation and were healthy when killed.

The ability of cells lacking PLD1 activity to produce a lethal infection was also determined using transgenic epsilon 26 (tge26) mice (Wang et al., 1994). Following oral inoculation, pld $1 \Delta 1$ mutant cells were significantly less virulent than wild-type strains of C. albicans (Table 2). Both wild-type strains tested (CAF2-1 and B311) were able to establish lethal infections resulting in mortality by $42 \mathrm{~d}$ post-challenge. In contrast, all mice inoculated with the PLD1-deficient yeast were alive and healthy at the end of the experiment. These assessments of virulence in two independent strains of immunodeficient mice indicate that the loss of PLD1 activity may have a profound effect on the ability of the cell to establish a disseminated and lethal infection.

\section{DISCUSSION}

One of the most prominent phenotypes of $S$. cerevisiae spo14 null mutants was the fact that cells were unable to sporulate (Ella et al., 1996; Rose et al., 1995; Waksman et al., 1996). Since C. albicans has not been shown to sporulate, we reasoned that a homologous phospholipase D1 in this fungus may have a different function. Earlier experiments from McLain \& Dolan (1997) showed that at least one calcium-independent, phosphatidylinositol 4,5-bisphosphate-stimulated, oleatesensitive form of PLD1 exists in C. albicans. These studies also demonstrated that this enzyme was important to the process of dimorphic transition. Research on the regulation of dimorphism was recently focused on MAP kinases, cAMP-regulated pathways and transcriptional regulators (Brown \& Gow, 1999; Ernst, 2000). In contrast, very few studies of the role of lipids as second messengers in the regulation of morphology in C. albicans have been reported (Gadd, 1995; Gadd \& Foster, 1997). Therefore, the aim of this study was to clone the PLD1 gene and to investigate the role and importance of this gene during growth and hyphal formation. The gene encoding PLD1 was cloned from a fosmid library. Sequencing of a major part of the ORF revealed a $98 \%$ identity to the sequence published by Kanoh et al. (1998). The observed differences may be due to sequencing errors, strain differences or the fact that different alleles were sequenced.

PLD1 is a large gene encoding a protein of 1710 aa with a calculated molecular mass of $196.4 \mathrm{kDa}$ (Kanoh et al., 1998). The overall identity of the deduced protein to ScPLD1 was 42\% (Kanoh et al., 1998) with highly conserved HXKXXXXD, HKD and GGGR motifs in the active site.

In this study, we found that PLD1 indeed showed a higher expression during the yeast to hyphae transition, but also constitutive expression during yeast growth in several media, indicating a more general function of this gene. To study the overall relevance of PLD1 for growth and during the dimorphic transition in particular, we designed pld1 null mutants that lacked an essential part of the ORF. Activity assays showed that these mutants were unable to produce PA, the hydrolytic product of PLD1, derived from PC indicating that PLD1 in fact encodes a phospholipase D. Moreover, these results suggested that PLD1 is the only or most prominent gene encoding a PC-specific PLD in C. albicans.

Null pld1 mutants were viable on all tested media, indicating that PLD1 is not an essential gene. Furthermore, no differences in growth rates compared to the wild-type were seen in all liquid media. These 
experiments included growth in protein medium, indicating that proteinase secretion was not significantly reduced. In addition, chlamydospore formation was still possible in the pld 1 mutants. In a model of oral infections, no attenuated virulence phenotype was observed. In two different mouse models, however, the pld1 11 mutant was significantly less virulent than wildtype strains.

Therefore, we concluded that PLD1 does not play a major role during growth, chlamydospore formation, secretion in liquid media or oral infections. However, on solid media marked differences were seen, with the lack of hyphal production on Spider medium the most prominent phenotype. Such an inability to form hyphae on Spider medium was also observed for the mutants lacking the transcriptional regulators Cph1 (Liu et al., 1994) or Efg1 (Stoldt et al., 1997). To prove that the observed phenotype was due to the lack of a functional PLD1 gene and not to undetected mutations introduced during the gene disruption procedure, we produced a second isogenic PLD1 null mutant ( $p l d 1 \Delta 2$ ). This second mutant showed the same phenotype as pld1 $1 \Delta 1$ under all conditions, making it unlikely that an undetected mutation was responsible for the observed phenotype.

In principle, PLD1 may act in two different ways during growth and dimorphism. It may produce lipid second messenger molecules which have regulatory functions or simply provide or change lipid molecules necessary as structural material for membranes (or both). For example, PA, LPA and DAG have been demonstrated to enhance membrane curvature (Kearns et al., 1997; Schmidt et al., 1999). Alternatively, PA may interact with proteins to alter cytoskeletal organization. PA has been demonstrated to stimulate the activity of phosphatidylinositol-4-phosphate 5-kinase in both mammals and S. cerevisiae; the resulting accumulation of phosphatidylinositol 4,5-bisphosphate can serve as a focal point for the assembly of cytoskeletal proteins containing pleckstrin homology (PH) domains (Lemmon et al., 1996; Tall et al., 1997). In addition, LPA may be generated by the concerted action of PLD1 and a phospholipase A (Mago \& Khuller, 1990; Goyal \& Khuller, 1992), which may act as an inducer of hyphal formation. LPA is known to be a major extracellular signal, produced in large amounts by activated platelets and other cells in human serum, which activates G proteins (Moolenaar, 1995; Gaits et al., 1997). Since a low molecular mass filtrate of serum was shown to be an inducing agent of the dimorphic transition via the RAS1 gene product (Feng et al., 1999), LPA may be a candidate signalling molecule which can be produced by host and/or Candida cells during infection. Finally, PLD1 may provide DAG, via lipid-phosphate phosphohydrolase, as a signalling molecule or material for a growing hyphal tip. In yeast cells, it has been shown that DAG plays an essential role in vesicular trafficking (Kearns et al., 1997).

A role for PLD1 activity in secretion is supported by work on phosphatidylinositol transfer proteins (PITPs) in C. albicans and other yeasts. The only published regulator protein of fungal PLD1 is an atypical PITP (Li et al., 2000). Although the physiological relevance of this regulation has not been established, this regulation adds to the genetic interaction described between PLD1 and the classic fungal PITP, Sec14p. SEC14 is an essential gene in S. cerevisiae and a number of extragenic 'SEC14 bypass' suppressors have been identified. For these suppressor mutations to bypass the requirement for Sec14, the cell must possess functional PLD1 (Sreenivas et al., 1998; Xie et al., 1998). This suppressor analysis suggests a role for PLD1 in secretion, particularly in the budding of nascent secretory vesicles from the transGolgi network. Furthermore, work on Sec14 in both Yarrowia lipolytica (Lopez et al., 1994) and C. albicans (Monteoliva et al., 1996; Riggle et al., 1997) has revealed a possible role for Sec14 in morphogenesis. Sec14 has been proposed to regulate the DAG pool necessary for proper secretory function (Kearns et al., 1997). Therefore, PLD1 might contribute to morphogenesis by ensuring that adequate levels of DAG are available for the secretion of components to the growing hyphal cell.

There are a number of possible important or even essential functions for PLD1. Nevertheless, disruption of the corresponding gene had only a minor effect. This may be due to a second PLD activity. However, our data suggest that a second PLD gene does exist or is expressed at very low levels. The fact that PLD1 is not an essential gene suggests that the cell is able to adapt to the loss of PLD1 activity by producing the necessary metabolites in different, PLD1-independent, ways. Since DAG is essential for viability, it is reasonable to propose that adequate levels of DAG can be produced by alternative pathways such as a phospholipase C. In fact, a gene encoding a phosphatidylinositol-specific phospholipase C (CAPLC1) has recently been cloned in C. albicans (Bennett et al., 1998). In addition to phospholipase C1derived DAG, DAG can be generated by the action of inositol-phosphoryl ceramide synthase (encoded by AUR1) which transfers phosphoinositol from phosphatidylinositol to phytoceramide with the release of DAG (Hashida-Okado et al., 1996). Another potential source for compensatory increases in DAG levels is dephosphorylation of PA generated by acylation of glycerol 3-phosphate (Athenstaedt \& Daum, 1997) instead of by hydrolysis of PC. Thus the cell has numerous mechanisms by which to generate DAG and PA, the two prominent products of PLD1 activity.

It has been reported recently that C. albicans may be capable of mating (Magee \& Magee, 2000; Hull et al., 2000). Such a capability would suggest that this organism is also capable of undergoing sporulation and meiosis. In fact, homologues of several S. cerevisiae genes involved in sporulation and meiosis have been identified. PLD1 is critical to the successful completion of meiosis and sporulation in S. cerevisiae. It will be interesting to determine whether homologues of sporulation regulators are able to alter the activity of PLD1 in C. albicans and whether PLD1 may be involved in sporulation of C. albicans.

The importance of lipid signalling in the metabolism 
and pathogenesis of fungi is only now becoming apparent. While the available information is very incomplete, it is already clear that lipid signalling has the potential to be as important to the cell as protein kinase signalling. Also of significance will be the manner in which cells respond to mutations that impact on the ability to utilize lipid messengers. Finally, the manner in which the cells are able to integrate the complex intracellular signals derived from so many different pathways and stimuli into a single coherent response represents a very rich area for future study.

\section{ACKNOWLEDGEMENTS}

We thank Dr S. Scherer, University of Minnesota, USA, for providing the fosmid library; Dr W. A. Fonzi, University of Washington, USA, for strains CAI4 and CAF2-1; Dr J. Ernst, University of Düsseldorf, Germany, for the efg1 mutant; Dr G. Fink, Massachusetts Institute of Technology, USA for the cph1 and efg1/cph1 mutants; Dr J. Gerst, Weizman Institute of Science, Israel, for the SPO14 gene; Dr E. Balish, Medical University of South Carolina, USA, for the mice data, and M. L. Hairfield, Medical University of South Carolina, USA, for the analysis of PLD1 activity in our mutants. This work was supported by the Deutsche Forschungsgemeinschaft (Grant Hu528/7-1) to B. H. and by MUSC Institutional Funds for Research to J.W.D.

\section{REFERENCES}

Athenstaedt, K. \& Daum, G. (1997). Biosynthesis of phosphatidic acid in lipid particles and endoplasmic reticulum of Saccharomyces cerevisiae. J Bacteriol 179, 7611-7616.

Bennett, D. E., McCreary, C. E. \& Coleman, D. C. (1998). Genetic characterization of a phospholipase $\mathrm{C}$ gene from Candida albicans: presence of homologous sequences in Candida species other than Candida albicans. Microbiology 144, 55-72.

Braun, B. R. \& Johnson, A. D. (1997). Control of filament formation in Candida albicans by the transcriptional repressor TUP1. Science 277, 105-109.

Brown, A. J. \& Gow, N. A. (1999). Regulatory networks controlling Candida albicans morphogenesis. Trends Microbiol 7, 333-338.

Buckley, H. R., Price, M. R. \& Daneo-Moore, L. (1982). Isolation of a variant of Candida albicans. Infect Immun 37, 1209-1217.

Buffo, J., Herman, M. A. \& Soll, D. R. (1984). A characterization of $\mathrm{pH}$-regulated dimorphism in Candida albicans. Mycopathologia 85, 21-30.

Colthurst, D. R., Schauder, B. S., Hayes, M. V. \& Tuite, M. F. (1992). Elongation factor 3 (EF-3) from Candida albicans shows both structural and functional similarity to EF-3 from Saccharomyces cerevisiae. Mol Microbiol 6, 1025-1033.

Cook, J. G., Bardwell, L., Kron, S. J. \& Thorner, J. (1996). Two novel targets of the MAP kinase Kss1 are negative regulators of invasive growth in the yeast Saccharomyces cerevisiae. Genes Dev 10, 2831-2848.

Ella, K. M., Dolan, J. W. \& Meier, K. E. (1995). Characterization of a regulated form of phospholipase D in the yeast Saccharomyces cerevisiae. Biochem J 307, 799-805.

Ella, K. M., Dolan, J. W., Qi, C. \& Meier, K. E. (1996). Characterization of Saccharomyces cerevisiae deficient in expression of phospholipase D. Biochem J 314, 15-19.

Ernst, J. (2000). Dimorphism in Candida albicans. In Contri- butions to Microbiology: Dimorphism in Human Pathogenic and Apathogenic Yeasts, pp. 98-111. Edited by J. F. Ernst \& A. Schmidt. Basel: Karger.

Feng, Q., Summers, E., Guo, B. \& Fink, G. (1999). Ras signaling is required for serum-induced hyphal differentiation in Candida albicans. J Bacteriol 181, 6339-6346.

Fodstad, Ø., Hansen, C. T., Cannon, G. B. \& Boyd, M. R. (1984). Immune characteristics of the beige-nude mouse: a model for studying immune surveillance. Scand J Immunol 20, 267-272.

Fonzi, W. A. \& Irwin, M. Y. (1993). Isogenic strain construction and gene mapping in Candida albicans. Genetics 134, 717-728.

Gadd, G. M. (1995). Signal transduction in fungi. In The Growing Fungus. Edited by N. A. R. Gow \& G. M. Gadd. London: Chapman \& Hall.

Gadd, G. M. \& Foster, S. A. (1997). Metabolism of inositol 1,4,5trisphosphate in Candida albicans: significance as a precursor of inositol polyphosphates and in signal transduction during the dimorphic transition from yeast cells to germ tubes. Microbiology 143, 437-448.

Gaits, F., Fourcade, O., Le Balle, F. \& 8 other authors (1997). Lysophosphatidic acid as a phospholipid mediator: pathways of synthesis. FEBS Lett 410, 54-58.

Gillum, A. M., Tsay, E. Y. H. \& Kirsch, D. R. (1984). Isolation of the Candida albicans gene for orotidine- $5^{\prime}$-phosphate decarboxylase by complementation of $S$. cerevisiae ura3 and E. coli pyrF mutations. Mol Gen Genet 198, 179-182.

Gow, N. A. \& Gooday, G. W. (1982). Vacuolation, branch production and linear growth of germ tubes in Candida albicans. J Gen Microbiol 128, 2195-2198.

Gow, N. A. R., Robbins, P. W., Brown, A. J. P., Fonzi, W. A., Chapman, T. \& Kinsman, O. K. (1994). A hyphal-specific chitin synthase gene (CHS2) is not essential for growth, dimorphism or virulence of Candida albicans. Proc Natl Acad Sci USA 91, 6216-6220.

Goyal, S. \& Khuller, G. K. (1992). Phospholipid composition and subcellular distribution in yeast and mycelial forms of Candida albicans. J Med Vet Mycol 30, 355-362.

Hashida-Okado, T., Ogawa, A., Endo, M., Yasumoto, R., Takesako, K. \& Kato, I. (1996). AUR1, a novel gene conferring aureobasidin resistance on Saccharomyces cerevisiae: a study of defective morphologies in Aur1p-depleted cells. Mol Gen Genet 251, 236-244.

Hube, B., Monod, M., Schofield, D. A., Brown, A. J. P. \& Gow, N. A. R. (1994). Expression of seven members of the gene family encoding secretory aspartyl proteinases in Candida albicans. Mol Microbiol 14, 87-99.

Hube, B., Sanglard, D., Odds, F. C., Hess, D., Monod, M., Schafer, W., Brown, A. J. \& Gow, N. A. (1997). Disruption of each of the secreted aspartyl proteinase genes SAP1, SAP2, and SAP3 of Candida albicans attenuates virulence. Infect Immun 65, 3529-3538.

Hube, B., Stehr, F., Bossenz, M., Mazur, A., Kretschmar, M. \& Schäfer, W. (2000). Secreted lipases of Candida albicans: cloning, characterisation and expression analysis of a new gene family with at least ten members. Arch Microbiol 174, 362-367.

Hull, C. M., Raisner, R. M. \& Johnson, A. D. (2000). Evidence for mating of the 'asexual' yeast Candida albicans in a mammalian host. Science 289, 307-310.

Kanoh, H., Nakashima, S., Zhao, Y., Sugiyama, Y., Kitajima, Y. \& Nozawa, Y. (1998). Molecular cloning of a gene encoding phospholipase D from the pathogenic and dimorphic fungus Candida albicans. Biochim Biophys Acta 1398, 359-364. 
Kearns, B. G., McGee, T. P., Mayinger, P., Gedvilaite, A., Phillips, S. E., Kagiwada, S. \& Bankaitis, V. A. (1997). Essential role for diacylglycerol in protein transport from the yeast Golgi complex. Nature 387, 101-105.

Lemmon, M. A., Ferguson, K. M. \& Schlessinger, J. (1996). PH domains: diverse sequences with a common fold recruit signaling molecules to the cell surface. Cell 85, 621-624.

Li, X., Routt, S. M., Xie, Z., Cui, X., Fang, M., Kearns, M. A., Bard, M., Kirsch, D. R. \& Bankaitis, V. A. (2000). Identification of a novel family of nonclassic yeast phosphatidylinositol transfer proteins whose function modulates phospholipase D activity and Sec14pindependent cell growth. Mol Biol Cell 11, 1989-2005.

Liu, H., Kohler, J. \& Fink, G. R. (1994). Suppression of hyphal formation in Candida albicans by mutation of a STE12 homolog. Science 266, 1723-1726.

Lopez, M. C., Nicaud, J. M., Skinner, H. B., Vergnolle, C., Kader, J. C., Bankaitis, V. A. \& Gaillardin, C. (1994). A phosphatidylinositol/phosphatidylcholine transfer protein is required for differentiation of the dimorphic yeast Yarrowia lipolytica from the yeast to the mycelial form. J Cell Biol 125, 113-127.

McLain, N. \& Dolan, J. W. (1997). Phospholipase D activity is required for dimorphic transition in Candida albicans. Microbiology 143, 3521-3526.

Magee, B. B. \& Magee, P. T. (2000). Induction of mating in Candida albicans by construction of MTLa and MTL $\alpha$ strains. Science 289, 310-313.

Magee, P. \& Scherer, S. (1998). Genome mapping and gene discovery in Candida albicans. ASM News 64, 505-511.

Mago, N. \& Khuller, G. K. (1990). Subcellular localization of enzymes of phospholipid metabolism in Candida albicans. J Med Vet Mycol 28, 355-362.

Maneu, V., Cervera, A. M., Martinez, J. P. \& Gozalbo, D. (1996). Molecular cloning and characterization of a Candida albicans gene (EFB1) coding for the elongation factor EF-1 $\beta$. FEMS Microbiol Lett 145, 157-162.

Mattia, E., Carruba, G., Angiolella, L. \& Cassone, A. (1982). Induction of germ tube formation by $\mathrm{N}$-acetyl-D-glucosamine in Candida albicans: uptake of inducer and germinative response. J Bacteriol 152, 555-562.

Mayr, J. A., Kohlwein, S. D. \& Paltauf, F. (1996). Identification of a novel, $\mathrm{Ca}(2+)$-dependent phospholipase $\mathrm{D}$ with preference for phosphatidylserine and phosphatidylethanolamine in Saccharomyces cerevisiae. FEBS Lett 393, 236-240.

Monteoliva, L., Sanchez, M., Pla, J., Gil, C. \& Nombela, C. (1996). Cloning of Candida albicans sec14 gene homologue coding for a putative essential function. Yeast 12, 1097-1105.

Moolennaar, W. H. (1995). Lysophosphatidic acid, a multifunctional phospholipid messenger. J Biol Chem 270, 12949-12952.

Morris, A. J., Frohman, M. A. \& Engebrecht, J. (1997). Measurement of phospholipase D activity. Anal Biochem 252, 1-9.

Riggle, P. J., Slobodkin, I. V., Brown, D. H., Jr, Hanson, M. P., Volkert, T. L. \& Kumamoto, C. A. (1997). Two transcripts, differing at their $3^{\prime}$ ends, are produced from the Candida albicans SEC14 gene. Microbiology 143, 3527-3535.

Roberts, R. L. \& Fink, G. R. (1994). Elements of a single MAP kinase cascade in Saccharomyces cerevisiae mediate two de- velopmental programs in the same cell type: mating and invasive growth. Genes Dev 8, 2974-2985.

Rose, K., Rudge, S. A., Frohman, M. A., Morris, A. J. \& Engebrecht, J. (1995). Phospholipase D signaling is essential for meiosis. Proc Natl Acad Sci U S A 92, 12151-12155.

Rudge, S. A., Morris, A. J. \& Engebrecht, J. (1998). Relocalization of phospholipase $\mathrm{D}$ activity mediates membrane formation during meiosis. J Cell Biol 140, 81-90.

Sambrook, J., Fritsch, E. F. \& Maniatis, T. (1989). Molecular Cloning: a Laboratory Manual, 2nd edn. Cold Spring Harbor, NY : Cold Spring Harbor Laboratory.

Schaller, M., Korting, H. C., Schäfer, W. \& Hube, B. (1998). Differential expression of secreted aspartyl proteinases in a model of human oral candidosis and in patient samples from the oral cavity. Mol Microbiol 29, 605-615.

Schmidt, A., Wolde, M., Thiele, C., Fest, W., Kratzin, H., Podtelejnikov, A. V., Witke, W., Huttner, W. B. \& Soling, H. D. (1999). Endophilin I mediates synaptic vesicle formation by transfer of arachidonate to lysophosphatidic acid. Nature 401, 133-141.

Sreenivas, A., Patton-Vogt, J. L., Bruno, V., Griac, P. \& Henry, S. A. (1998). A role for phospholipase D (Pld1p) in growth, secretion, and regulation of membrane lipid synthesis in yeast. J Biol Chem 273, 16635-16638.

Stoldt, V. R., Sonneborn, A., Leuker, C. E. \& Ernst, J. F. (1997). $\mathrm{Efg} 1 \mathrm{p}$, an essential regulator of morphogenesis of the human pathogen Candida albicans, is a member of a conserved class of bHLH proteins regulating morphogenetic processes in fungi. EMBO J 16, 1982-1991.

Tall, E., Dorman, G., Garcia, P. \& 8 other authors (1997). Phosphoinositide binding specificity among phospholipase C isozymes as determined by photo-cross-linking to novel substrate and product analogs. Biochemistry 36, 7239-7248.

Vasquez-Torres, A., Jones-Carson, J., Wagner, R. D., Warner, T. \& Balish, E. (1999). Early resistance of interleukin-10 knockout mice to acute systemic candidiasis. Infect Immun 67, 670-674.

Waksman, M., Eli, Y., Liscovitch, M. \& Gerst, J. E. (1996). Identification and characterization of a gene encoding phospholipase D activity in yeast. J Biol Chem 271, 2361-2364.

Waksman, M., Tang, X., Eli, Y., Gerst, J. E. \& Liscovitch, M. (1997). Identification of a novel $\mathrm{Ca}^{2+}$-dependent, phosphatidylethanolamine-hydrolyzing phospholipase D in yeast bearing a disruption in PLD1. J Biol Chem 272, 36-39.

Wang, B., Biron, C., She, J., Higgins, K., Sunshine, M. J., Lacy, E., Lonberg, N. \& Terhorst, C. (1994). A block in both early $T$ lymphocyte and natural killer cell development in transgenic mice with high-copy numbers of the human CD3E gene. Proc Natl Acad Sci US A 91, 9402-9406.

Xie, Z., Fang, M., Rivas, M. P., Faulkner, A. J, Sternweis, P. C., Engebrecht, J. A. \& Bankaitis, V. A. (1998). Phospholipase D activity is required for suppression of yeast phosphatidylinositol transfer protein defects. Proc Natl Acad Sci USA 95, 12346-12351.

Received 13 June 2000; revised 27 November 2000; accepted 22 December 2000. 